\title{
On the Dynamics of the 4d Spin Glass in a magnetic field
}

\author{
Giorgio Parisi, Federico Ricci-Tersenghi \\ and Juan J. Ruiz-Lorenzo \\ Dipartimento di Fisica and INFN, Università di Roma La Sapienza \\ P. A. Moro 2, 00185 Roma (Italy) \\ giorgio.parisi@roma1.infn.it \\ riccife@chimera.roma1.infn.it \\ ruiz@chimera.roma1.infn.it
}

November 13, 1997

\begin{abstract}
We study the four dimensional Gaussian spin glass in presence of a magnetic field. Using off-equilibrium numerical simulations we have found that the probability distribution of the overlaps is built in the same way as that of the Mean Field approximation with replica symmetry breaking. Finally we have studied the violation of the fluctuation-dissipation theorem in presence of magnetic field.
\end{abstract}




\section{Introduction}

One of the main open questions in the study of finite dimensional spin glasses is the existence of a phase transition in presence of an external field. While in absence of magnetic field the droplet model [1] and the Mean Field (MF) picture [2] both predict the existence of a phase transition, in presence of magnetic field the situation is fully different: Mean Field predicts a phase transition whereas the droplet model shows that the magnetic field destroys the frozen phase.

Moreover there are some analytical arguments which imply that the phase transition in presence of magnetic field is of a rather peculiar type. For instance Bray and Roberts [3], working with a reduced theory (obtained by projecting the original theory in the replicon subspace; in presence of magnetic field that is the only critical mode), have shown that there is no weak-coupling fixed point in magnetic field in their renormalization group equations also near 6 dimensions. An absence of a weak-coupling fixed point is often taken as an indication of a first order transition. Here the situation is quite less clear.

The very existence of a transition is still controversial and not too much work has been devoted to its study. Numerical simulations have been done in the past [1]. They were compatible with the possibility of a transition, but the situation was not so clearly cut and no convincing conclusions could be reached. Only recently an off-equilibrium numerical simulations in 4 dimensions have strongly suggested the existence of a phase transition in presence of magnetic field [5].

Two main advantages in using dynamical methods are that we can simulate very large systems (up to $40^{4}$ in this work) loosing practically all the finite size effects and that they are quicker with respect to an equilibrium simulation, because we don't need to thermalize. These methods have been already largely used in the numerical studies of spin glasses (see for instance reference [6]).

In the broken replica symmetry solution of the SK model [2, [7] there is a phase transition also when the system is plunged into a magnetic field and the line $T_{\mathrm{c}}(h)$ which separates the paramagnetic from the spin glass phase is called Almeida-Thouless (AT) line [8]. The order parameter of the Mean Field theory is the probability distribution of the overlaps, $P(q)$.

In absence of external field $P(q)$ is a delta function centered on $q=0$ for $T>T_{\mathrm{c}}$, while for $T<T_{\mathrm{c}}$ it becomes a highly non trivial function with two delta functions on the values

$q= \pm q_{\mathrm{EA}}\left(q_{\mathrm{EA}}\right.$ is the maximum allowed value for the overlap) and a non-zero part between them.

When a magnetic field is switched on the function $P(q)$ becomes zero for every negative overlap and the minimum allowed value for $q$ is shifted upwards from $-q_{\mathrm{EA}}$ to $q_{\min }>0$, while the maximum value $\left(q_{\mathrm{EA}}\right)$ almost doesn't change. This means, in terms of the distribution function of the overlaps, that for $T>T_{\mathrm{c}}(h)$ the function $P(q)$ is a delta function centered on a strictly positive value and that for $T<T_{\mathrm{c}}(h)$ the function $P(q)$ is the sum of a delta function on the maximum value $q_{\max }=q_{\text {EA }}$ plus a non-zero part down to $q_{\min }>0$ and a smaller weighted delta function on $q_{\min }$.

In this paper we present evidences for a Mean Field like phase transition at finite temperature. We show that the order parameter $P(q)$ has a non-zero support: we have characterized numerically the mean, the minimum and the maximum value allowed, de- 
noted $\bar{q}, q_{\min }$ and $q_{\max }$ respectively, and we have found that $q_{\min }<\bar{q}<q_{\max }$.

The plan of the paper is the following. In the next section we fix the notation and we describe the quantities we have measured. In sections three we show the numerical results. Finally we present the conclusions.

\section{The model and the observables}

We have simulated the Gaussian Ising spin glass in four dimensions on a hypercubic lattice of volume $N=L^{4}$ with periodic boundary conditions. The Hamiltonian of the system is given by

$$
\mathcal{H}=-\sum_{<i j>} \sigma_{i} J_{i j} \sigma_{j}-\sum_{i} \sigma_{i} h_{i}
$$

By $\left\langle i j>\right.$ we denote the sum over nearest neighbor pairs. The $J_{i j}$ are Gaussian variables with zero mean and unitary variance. The external field is also Gaussian with zero mean and variance $h_{0}^{2}$. We have studied systems with $h_{0}=0.05,0.1,0.2,0.3,0.5$.

We can justify the choice of a Gaussian magnetic field as follows. The starting point is the Ising spin glass Hamiltonian with an uniform magnetic field $h_{0}$

$$
\mathcal{H}_{0}=-\sum_{<i j>} \sigma_{i} J_{i j} \sigma_{j}-h_{0} \sum_{i} \sigma_{i}
$$

We can perform the following transformation (a "local gauge transformation") on the couplings: $J_{i j} \rightarrow J_{i j}^{\prime} \equiv n_{i} J_{i j} n_{j}$, where $n_{i}=+1$ or -1 . This transformation leaves the Hamiltonian $\mathcal{H}_{0}$ unchanged because the probability distribution of the couplings is Gaus$\operatorname{sian}\left(J_{i j}^{2}=\left(J_{i j}^{\prime}\right)^{2}\right)$. Now we recast the spins to $s_{i} \equiv n_{i} \sigma_{i}$, and finally our Hamiltonian reads

$$
\mathcal{H}_{0}=-\sum_{<i j>} s_{i} J_{i j} s_{j}-\sum_{i}\left(h_{0} n_{i}^{\prime}\right) s_{i}
$$

where $n_{i}^{\prime} n_{i}=1$. We remark the full arbitrariness of the choice of $n_{i}^{\prime}$ 's. In particular we can choose them from a bimodal distribution: i.e. $n_{i}^{\prime}=1$ with probability $1 / 2$ and -1 with the same probability. And so, if we define $h_{i}^{\prime} \equiv h_{0} n_{i}^{\prime}$, the Hamiltonian can be written as

$$
\mathcal{H}_{0}=-\sum_{<i j>} s_{i} J_{i j} s_{j}-\sum_{i} h_{i}^{\prime} s_{i}
$$

We have therefore shown that a spin glass with an uniform magnetic field $\left(h_{0}\right)$ is equivalent to a spin glass in which the magnetic field is random with zero mean and variance $h_{0}^{2}$. The probability distribution of such a magnetic field is bimodal, not Gaussian. Nevertheless there are not reasons to suppose a different physical behavior of these two cases (bimodal and Gaussian). We have chosen a Gaussian distribution, and not a bimodal one, because in the Gaussian case there are exact relations among some quantities.

We are interested in measuring the mean value of the overlap between two replicas, which is defined as $\bar{q}=\int q P(q) \mathrm{d} q$, without doing expensive equilibrium simulations. If we take two replicas in random configurations (as we do at the beggining of a simulation) their overlap is zero $(q(t=0)=0)$. Letting them evolve, the overlap will never increase 
beyond $q_{\text {min }}$, defined as the minimum overlap allowed at the equilibrium 円. This fact has been largely verified also in off-equilibrium simulations without magnetic field: during the simulation the overlap fluctuates around zero or slightly grows. This observation gives us a practical tool to calculate $q_{\text {min }}$ via an infinite-time extrapolation, but also asserts that we cannot get information on the whole $P(q)$ simply by looking at the off-equilibrium overlap.

To measure $\bar{q}$ we have exploited a relation valid at equilibrium when the applied field is Gaussian, which reads

$$
\frac{\overline{\left\langle\sigma_{i} h_{i}\right\rangle}}{h_{0}^{2}}=\frac{1-\bar{q}}{T}
$$

where with the overline we mean an average over the quenched random interactions and external fields. This relation can be easily obtained via an integration by parts (exploiting that $h_{i}$ is a Gaussian random variable) and it's exact also in a finite volume. In order to compute $\bar{q}$ we can measure $\overline{\left\langle\sigma_{i}(t) h_{i}\right\rangle}$, which is a quantity that rapidly converges to its infinite-time value. The fact that $q_{\text {min }}$ differs from $\bar{q}$ is a clear signal of replica symmetry breaking.

The second part of our study is focused on the fluctuation-dissipation theorem (FDT) and its generalization in the out of equilibrium regime [10, 11, 12, called off-equilibrium fluctuation-dissipation relation (OFDR). In reference [12] a detailed study of such a relation in finite dimensional spin glasses without magnetic field can be found. Here we extend those studies in presence of a magnetic field, obtaining similar results, which confirm the Mean Field behavior of the phase transition.

To study the OFDR we have measured the spin-spin autocorrelation function, $C\left(t, t_{w}\right)$, and the integrated response of the system, $\overline{\left\langle\sigma_{i} \tilde{h}_{i}\right\rangle} / \epsilon_{0}^{2}$, where the perturbation to the Hamiltonian $\mathcal{H}$ (eq.(1)), $\tilde{h}$, is a random Gaussian magnetic field with zero mean and variance $\epsilon_{0}^{2}$. In the next paragraphs we will obtain a formula that links, even in the early times of the dynamics, the response and the auto-correlation function.

Given a quantity $A(t)$ that depends on the local variables of our original Hamiltonian $(\mathcal{H})$. We can define the associate autocorrelation function

$$
C\left(t, t^{\prime}\right) \equiv\left\langle A(t) A\left(t^{\prime}\right)\right\rangle
$$

and the response function

$$
\left.R\left(t, t^{\prime}\right) \equiv \frac{\delta\langle A(t)\rangle}{\delta \epsilon\left(t^{\prime}\right)}\right|_{\epsilon=0},
$$

where we have assumed that the original Hamiltonian has been perturbed by a term

$$
\mathcal{H}^{\prime}=\mathcal{H}+\int \epsilon(t) A(t) \mathrm{d} t
$$

The brackets $\langle(\cdots)\rangle$ in eq.(6) and eq.(7) imply here a double average, one over the dynamical process and a second over the disorder.

\footnotetext{
${ }^{1}$ This can be better understood if we think that $P(q)$ is the equilibrium distribution of a dynamical variable which feels a potential $V(q)=-\log P(q)$. The peak at $q=q_{\text {min }}$ in $P(q)$ corresponds to a deep well in $V(q)$ and if $q$ starts with a value less than $q_{\min }$ it will go downhill towards $q_{\min }$. See reference [9] for more details.
} 
In the dynamical framework assuming time translational invariance it is possible to derive the fluctuation-dissipation theorem, that reads

$$
R\left(t, t^{\prime}\right)=\beta \theta\left(t-t^{\prime}\right) \frac{\partial C\left(t, t^{\prime}\right)}{\partial t^{\prime}}
$$

In spin models a common choice for $A(t)$ is $A(t)=\sigma_{i}(t)$ or $A(t)=N^{-1 / 2} \sum_{i} \sigma_{i}(t)$. In this case, because the system feels a magnetic field, to have a simpler response we should perturb it with a random field, $\tilde{h}$, and measure the staggered magnetization. In order to derive a fluctuation theorem where the response is related to the one site correlation, we must chose a perturbation such that the off-site elements of the response are zero 7 . So here we put $A(t)=N^{-1 / 2} \sum_{i} n_{i} \sigma_{i}(t)$, where $n_{i}=\tilde{h}_{i} / \epsilon_{0}$. Thanks to the fact that $\left\langle n_{i} n_{j}\right\rangle=\delta_{i, j}$, we have that with this choice

$$
C\left(t, t^{\prime}\right)=\frac{1}{N} \sum_{i}\left\langle\sigma_{i}(t) \sigma_{i}\left(t^{\prime}\right)\right\rangle
$$

and

$$
R\left(t, t^{\prime}\right)=\frac{\delta m_{\mathrm{s}}[\tilde{h}](t)}{\delta \tilde{h}\left(t^{\prime}\right)}
$$

where

$$
m_{\mathrm{s}}[\tilde{h}](t)=\frac{1}{N} \sum_{i}\left\langle n_{i} \sigma_{i}(t)\right\rangle
$$

is the staggered magnetization, which is a functional of the magnetic field, $\tilde{h}(t)$, and a function of the time.

The fluctuation-dissipation theorem holds in the equilibrium regime, but in the early times of the dynamics we expect a breakdown of its validity. Mean Field studies [13\| suggest the following modification of the FDT:

$$
R\left(t, t^{\prime}\right)=\beta X\left(t, t^{\prime}\right) \theta\left(t-t^{\prime}\right) \frac{\partial C\left(t, t^{\prime}\right)}{\partial t^{\prime}}
$$

It has also been suggested in [13, 14, 15] that the function $X\left(t, t^{\prime}\right)$ is only a function of the autocorrelation: $X\left(t, t^{\prime}\right)=X\left(C\left(t, t^{\prime}\right)\right)$. We can then write the following generalization of FDT, which should hold in early times of the dynamics, the off-equilibrium fluctuationdissipation relation (OFDR), that reads

$$
R\left(t, t^{\prime}\right)=\beta X\left(C\left(t, t^{\prime}\right)\right) \theta\left(t-t^{\prime}\right) \frac{\partial C\left(t, t^{\prime}\right)}{\partial t^{\prime}} .
$$

We can use the previous formula, eq.(14), to relate the observable quantities defined in eq.(10) and eq.(12). Using the functional Taylor expansion we can write

$$
m_{\mathrm{s}}[\tilde{h}](t)=m_{\mathrm{s}}[0](t)+\left.\int_{-\infty}^{\infty} \mathrm{d} t^{\prime} \frac{\delta m_{\mathrm{s}}[\tilde{h}](t)}{\delta \tilde{h}\left(t^{\prime}\right)}\right|_{\tilde{h}(t)=0} \tilde{h}\left(t^{\prime}\right)+\mathrm{O}\left(\tilde{h}^{2}\right) .
$$

\footnotetext{
${ }^{2}$ We notice also that in absence of a magnetic fields, a constant field perturbation is gauge equivalent to a random magnetic field.
} 
Exploiting the definition of eq.(11) and using the fact that $m[0](t)=0$ for every perturbing field orthogonal to the pre-existing one, i.e. such that $\sum_{i}\left\langle n_{i} h_{i}\right\rangle=0$ (which is true if $h_{i}$ is another (uncorrelated) random field, as happens in our case), we obtain

$$
m_{\mathrm{s}}[\tilde{h}](t)=\int_{-\infty}^{t} \mathrm{~d} t^{\prime} R\left(t, t^{\prime}\right) \tilde{h}\left(t^{\prime}\right)+\mathrm{O}\left(\tilde{h}^{2}\right) .
$$

This is just the linear-response theorem neglecting higher orders in $\tilde{h}$.

By applying the OFDR we obtain the dependence of the staggered magnetization with time in a generic time-dependent magnetic field (with a small strength), $\tilde{h}(t)$. [?

$$
m_{\mathrm{s}}[\tilde{h}](t) \simeq \beta \int_{-\infty}^{t} \mathrm{~d} t^{\prime} X\left[C\left(t, t^{\prime}\right)\right] \frac{\partial C\left(t, t^{\prime}\right)}{\partial t^{\prime}} \tilde{h}\left(t^{\prime}\right) .
$$

Now we can perform the following experiment. We let the system evolve with the unperturbed Hamiltonian of eq.(1) from $t=0$ to $t=t_{w}$, and then we turn on the perturbing magnetic field $\tilde{h}$, which is Gaussian distributed with zero mean and time-independent variance, $\epsilon_{0}^{2}$. Finally, with this choice of the magnetic field, we can writef

$$
m_{\mathrm{s}}[\tilde{h}](t) \simeq \epsilon_{0} \beta \int_{t_{w}}^{t} \mathrm{~d} t^{\prime} X\left[C\left(t, t^{\prime}\right)\right] \frac{\partial C\left(t, t^{\prime}\right)}{\partial t^{\prime}},
$$

and by performing the change of variables $u=C\left(t, t^{\prime}\right)$, equation (18) reads

$$
m_{\mathrm{s}}[\tilde{h}](t) \simeq \epsilon_{0} \beta \int_{C\left(t, t_{w}\right)}^{1} \mathrm{~d} u X[u],
$$

where we have used the fact that $C(t, t) \equiv 1$ (always true for Ising spins). In the equilibrium regime (FDT holds, $X=1$ ) we must obtain

$$
m_{\mathrm{s}}[\tilde{h}](t) \simeq \epsilon_{0} \beta\left(1-C\left(t, t_{w}\right)\right),
$$

i.e. $m_{\mathrm{s}}[\tilde{h}](t) T / \epsilon_{0}$ is a linear function of $C\left(t, t_{w}\right)$ with slope -1 . We remark that we can use this formula to obtain $q_{\max }$ as the point where the curve $m_{\mathrm{s}}[\tilde{h}](t)$ versus $C\left(t, t_{w}\right)$ leaves the line with slope $-\beta \epsilon_{0}$ (as we will explain).

In the limit $t, t_{w} \rightarrow \infty$ with $C\left(t, t_{w}\right)=q$, one has that $X(C) \rightarrow x(q)$, where $x(q)$ is given by

$$
x(q)=\int_{q_{\min }}^{q} \mathrm{~d} q^{\prime} P\left(q^{\prime}\right),
$$

where $P(q)$ is the equilibrium probability distribution of the overlap. Obviously $x(q)$ is equal to 1 for all $q>q_{\max }$, and we recover FDT for $C\left(t, t_{w}\right)>q_{\max }$. This link between the dynamical function $X(C)$ and the static one $x(q)$ has been already verified for finite dimensional spin glasses [12.

For future convenience, we define

$$
S(C) \equiv \int_{C}^{1} \mathrm{~d} q x(q)=\int_{C}^{1} \mathrm{~d} q \int_{q_{\min }}^{q} \mathrm{~d} q^{\prime} P\left(q^{\prime}\right) .
$$

\footnotetext{
${ }^{3}$ The symbol $\simeq$ means that the equation is valid in the region where linear-response holds.

${ }^{4}$ We ignore in our notation the fact that $m_{\mathrm{s}}[\tilde{h}](t)$ depends also on $t_{w}$.
} 
or equivalently

$$
P(q)=-\left.\frac{\mathrm{d}^{2} S(C)}{\mathrm{d}^{2} C}\right|_{C=q} .
$$

In the limit where $X \rightarrow x$ we can write eq.(19) as

$$
\frac{m_{\mathrm{s}}[\tilde{h}](t) T}{\epsilon_{0}} \simeq S\left(C\left(t, t_{w}\right)\right) .
$$

Looking at the relation between the correlation function and the integrated response function for large $t_{w}$ we can thus obtain $q_{\max }$, the maximum overlap with non-zero $P(q)$, as the point where the function $S(C)$ becomes different from the function $1-C$, and $q_{\min }$ as the smallest value of $C$.

At this point we have numerical methods to compute three important different values of $q: q_{\max }, q_{\min }$ and $\bar{q}$.

\section{$3 \quad$ Numerical results}

\section{$3.1 \quad \bar{q}$ and $q_{\min }$}

We are interested in the behavior of the system in the out of equilibrium regime, so we do not need to thermalize the sample and we can simulate very large samples of millions of spins $\left(24^{4}, 32^{4}\right.$ and $\left.40^{4}\right)$. We expect our data not to be affected by large finite size bias: we find that, in the range of temperature considered, the data for different lattice sizes ( $L=24,32,40)$ coincide within the error-bars, with the largest systems $(L=32,40)$ giving practically the same values.

All the numerical simulations have been performed on the parallel super-computer APE100 [16].

In the first part of our study we have done simulations using the Hamiltonian of eq.(位) without perturbing the system $(\tilde{h}=0)$.

According to our dynamical approach, we are interested in doing measurements in the off-equilibrium regime of large times. So we perform the simulations following an annealing schedule, with slower and slower cooling rates. This is equivalent to run a simulation at a fixed temperature, considering larger and larger waiting times [12, 17]. The advantage is twice: within a single run we are able to collect data at different temperatures and there are smaller finite-time effects because when the temperature is lowered by an amount $\Delta T$ the time needed to "forget" the previous temperature is smaller than if it had been quenched from $T=\infty$. In the present case the range of temperatures $\mathrm{P}$ is from $T=3.0$ down to $T=0.5$ with a step of $\Delta T=0.25$. The cooling rates have been chosen in such a way that in the $s$-th annealing run the number of Monte Carlo Steps (MCS) is proportional to $2^{s}$, with $s$ ranging from 0 to 12 . The annealing procedure is the following: indexing the temperatures from the highest $\left(i_{T=3.0}=1\right)$ to the lowest $\left(i_{T=0.5}=11\right)$ the number of MCS at each temperature is $2^{s} \cdot i_{T}^{2}$. This means that in the slowest run the system will stay at the lowest temperature for about half a million MCS.

\footnotetext{
${ }^{5}$ We remind that in the four dimensional Gaussian Ising spin glass without external field the transition temperature is $T_{\mathrm{c}}=1.8$ [17.
} 


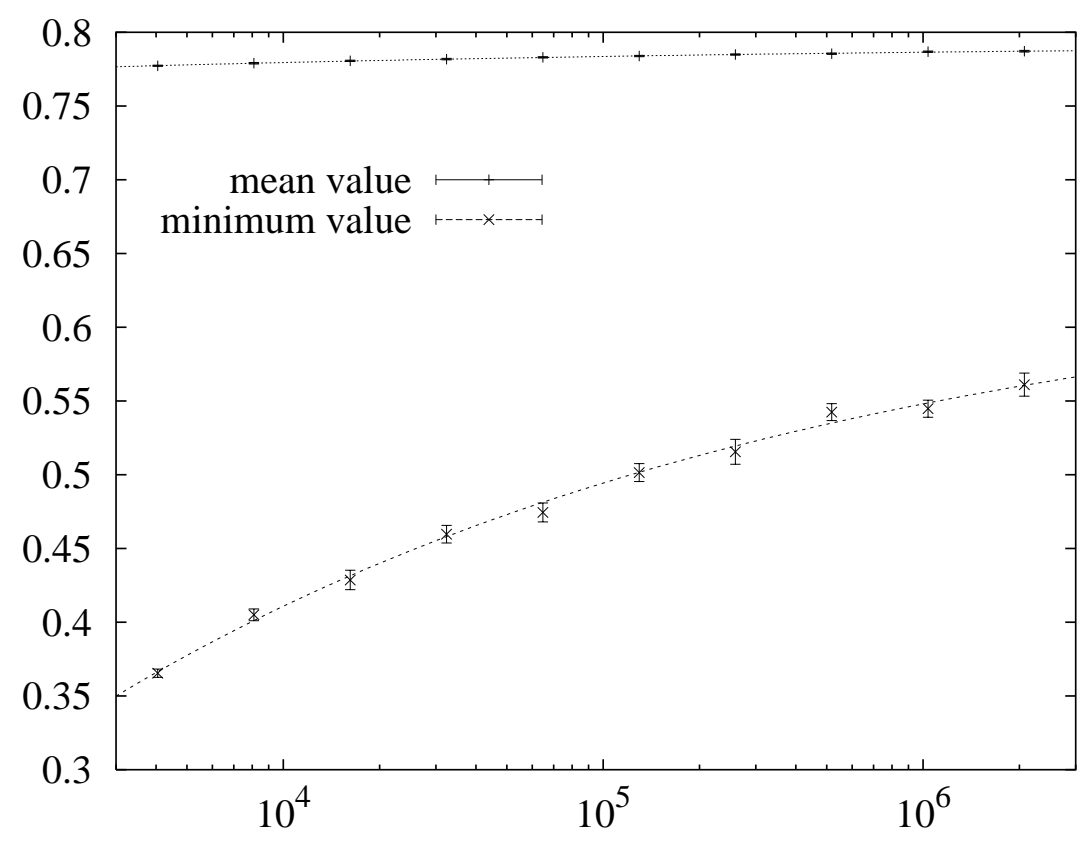

Figure 1: Data of $q(t)$ (bottom) and $1-\overline{\left\langle\sigma_{i}(t) h_{i}\right\rangle} T / h_{0}^{2}$ (top) versus Monte Carlo time. The curves are the best fits (see text). $L=40, h_{0}=0.3$ and $T=0.5$.

To analyze the data, we fix a temperature, we recollect the data at that temperature from different annealing runs and we extrapolate the result in the limit of infinitely slow cooling. This will give us information on the large times off-equilibrium regime.

In particular we look at the overlap between two replicas, $q(t)$, which start very far from each other: $q(t=0)=0$. In the limit $t \rightarrow \infty$ this overlap will tend to the minimum overlap allowed at the equilibrium, $q_{\min }$. Also we have measured $\overline{\left\langle\sigma_{i}(t) h_{i}\right\rangle}$ from which we obtain the value of $\bar{q}$ using eq.(5). In figure 1 we show the data $\left(L=40, h_{0}=0.3\right.$ and $T=0.5)$ whose infinite-time extrapolation gives the mean and the minimum value for the overlap (top and bottom data respectively).

We fit the data plotted in figure 1 with the following formula: $q(t)=A t^{-B}+C$. If the value of the exponent $B$ were too small the uncertainty on the value of $C$ should be very large and the significance of the fit very poor. We have found that it decreases with decreasing temperature or magnetic field and we have checked in all our fits that it were not too small. In figure 1 we present the worst case we can fit satisfactory (lowest temperature and $\left.h_{0}=0.3\right)$, obtaining as the best parameters: $A=-0.069(2), B=0.20(3)$ and $C=\bar{q}=0.792(5)$ for the top data and $A=-1.4(2), B=0.20(3)$ and $C=q_{\text {min }}=0.64(2)$ for the bottom data. Both fits have very good $\chi^{2}$ values. Note that the best $B$ exponent is the same in both fits. The presence of a power law approach to equilibrium can be associated to the existence of a well defined off-equilibrium correlation length in finite dimensional spin glasses which grows with a power law of the time [17, 18, 19].

The dynamical approach in presence of a magnetic field has the advantage that, using a fitting function of the type $A t^{-B}+C$ for any observable in the off-equilibrium regime, the 


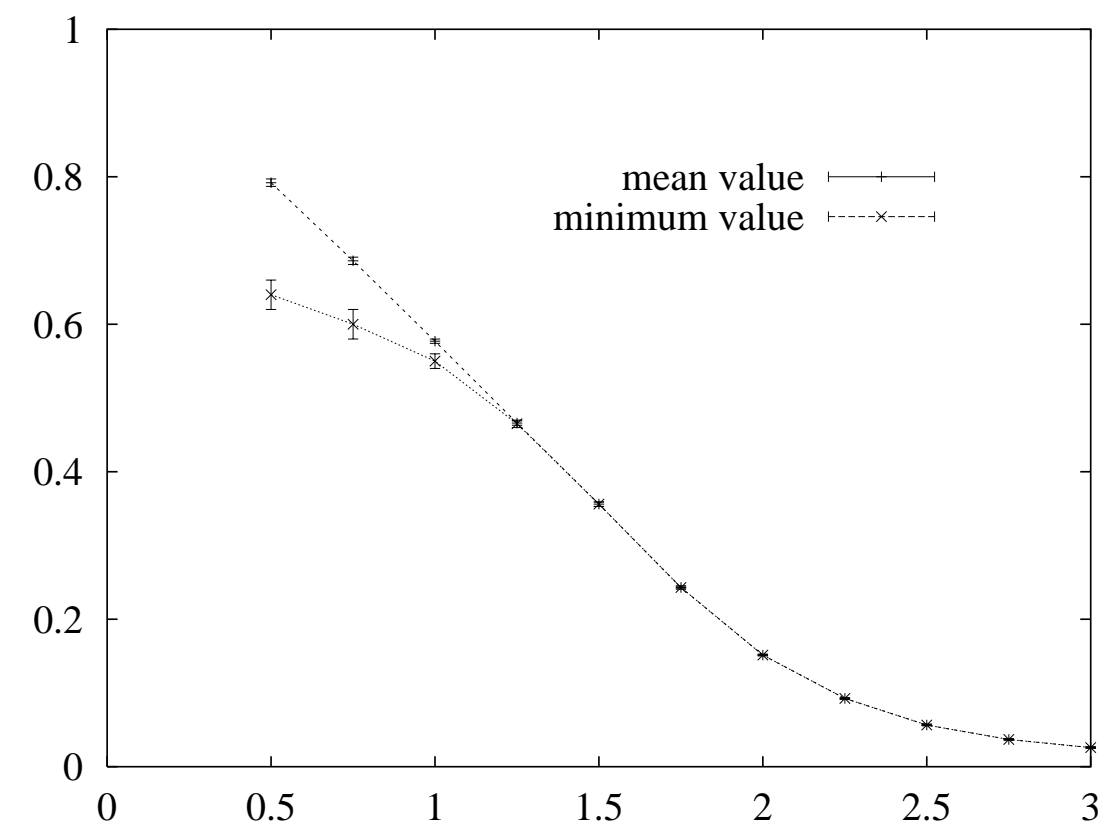

Figure 2: $\bar{q}$ (top) and $q_{\min }$ (bottom) versus $T$ for $L=40$ and $h_{0}=0.3$.

best value for the exponent $B$ is always greater than in the case of exactly zero external field and moreover it has a finite limit when $T \rightarrow 0$, while for $h=0$ very often $B \propto T$. The reasons for this difference in behaviour are unclear to us $[$.

Now we clearly see the usefulness of the trick of using a Gaussian magnetic field: we can calculate $\bar{q}$ from the extrapolation of $\overline{\left\langle\sigma_{i}(t) h_{i}\right\rangle}$, which is a practically constant quantity with respect to $q(t)$ (look at figure 1). Moreover we don't need to do any limit of small $h$ and so we don't have the problem of very small $B$ exponents.

The same kind of fitting analysis has been done for all temperatures, obtaining values for $q_{\min }(T)$ and $\bar{q}(T)$, plotted in figure 2. In the high temperature phase the extrapolated values for $q_{\min }$ and $\bar{q}$ coincide (as they should because the equilibrium overlap distribution function is a delta function) confirming the correctness of the dynamical method.

Figure 2 gives clear evidence of a wide region $\left(T<T_{\mathrm{c}}\left(h_{0}=0.3\right) \simeq 1.2\right)$ where the order parameter $P(q)$ is not a single delta function. We have calculated the functions $q_{\min }(T)$ and $\bar{q}(T)$ also for different values of the magnetic field. We see a clear bifurcation also for $h_{0}=0.5$ at a temperature $T_{\mathrm{c}}\left(h_{0}=0.5\right) \simeq 1.0$, while for lower magnetic fields the results are less clean because the errors are larger (due to the just described problem in the extrapolation procedure).

The last result we present in this section is the shape of the equilibrium magnetization in a magnetic field as a function of the temperature. More precisely what we show in fig 3

\footnotetext{
${ }^{6}$ We would like to recall that numerical simulations of relative small size systems for the SK model indicate that the time for passing at equilibrium from one value of $q$ to an other value of $q$ with the same sign increases as $\exp \left(A N^{1 / 4}\right)$ while that for changing the sign of $q$ increases as $\exp \left(A N^{1 / 2}\right)$. In magnetic field only the first processes are present and in the SK model they correspond to much faster movements.
} 


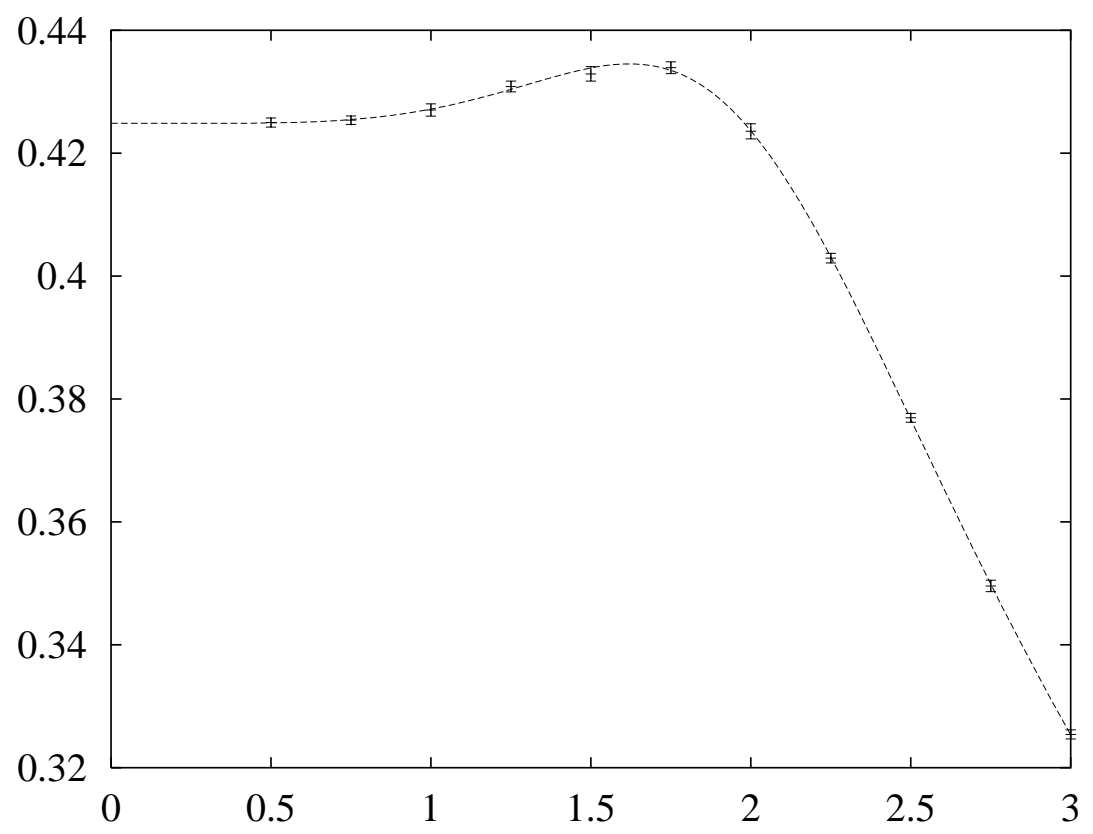

Figure 3: $\overline{\left\langle\sigma_{i} h_{i}\right\rangle} / h_{0}^{2}$ versus $T$ for $L=40$ and $h_{0}=0.3$. The curve is only a guide to the eye.

is the staggered magnetization $\overline{\left\langle\sigma_{i} h_{i}\right\rangle} / h_{0}^{2}$, but we have verified that it behaves qualitatively and also almost quantitatively like the magnetization in a constant field.

We observe a behavior similar to the one measured in experiments on real samples: the magnetization has a little peak, whose height is greater than the zero-temperature value by a few percents. We want to stress that the peak is at a temperature higher than the critical one, so the magnetization peak is not exactly on the AT-line.

\subsection{Fluctuation-Dissipation in presence of magnetic field}

In the second part of our study we have simulated an Ising spin glass in a Gaussian magnetic field with variance $h_{0}^{2}\left(h_{0}=0.2,0.3,0.5\right)$ at a fixed temperature $(T=0.75,1.0,1.5)$. We have measured, for various waiting times $\left(t_{w}=2^{8}, 2^{11}, 2^{14}, 2^{17}\right)$, the autocorrelation function, defined in eq.(10), and the staggered magnetization, defined in eq.(12), with different amplitudes of the perturbing field $\left(\epsilon_{0}=0.02,0.03,0.06\right)$. We are interested in the relation between these two quantities, which tends to the function $X(C)$ in the large $t_{w}$ limit (see eq.(24)). The data do not depend on the choice of $\epsilon_{0}$ or $L$, so the results we will show are in the linear-response regime and they are not affected by large finite size biases. In these runs the starting configuration of the spins is always random.

We can keep in mind the possible link between the static and dynamics (that we do not study in the present paper) for a spin glass in presence of a magnetic field and we can compute the qualitative form of the function $X(C)$ using as input the $S(C)$ function obtained in the Mean Field approximation and in the droplet model. 


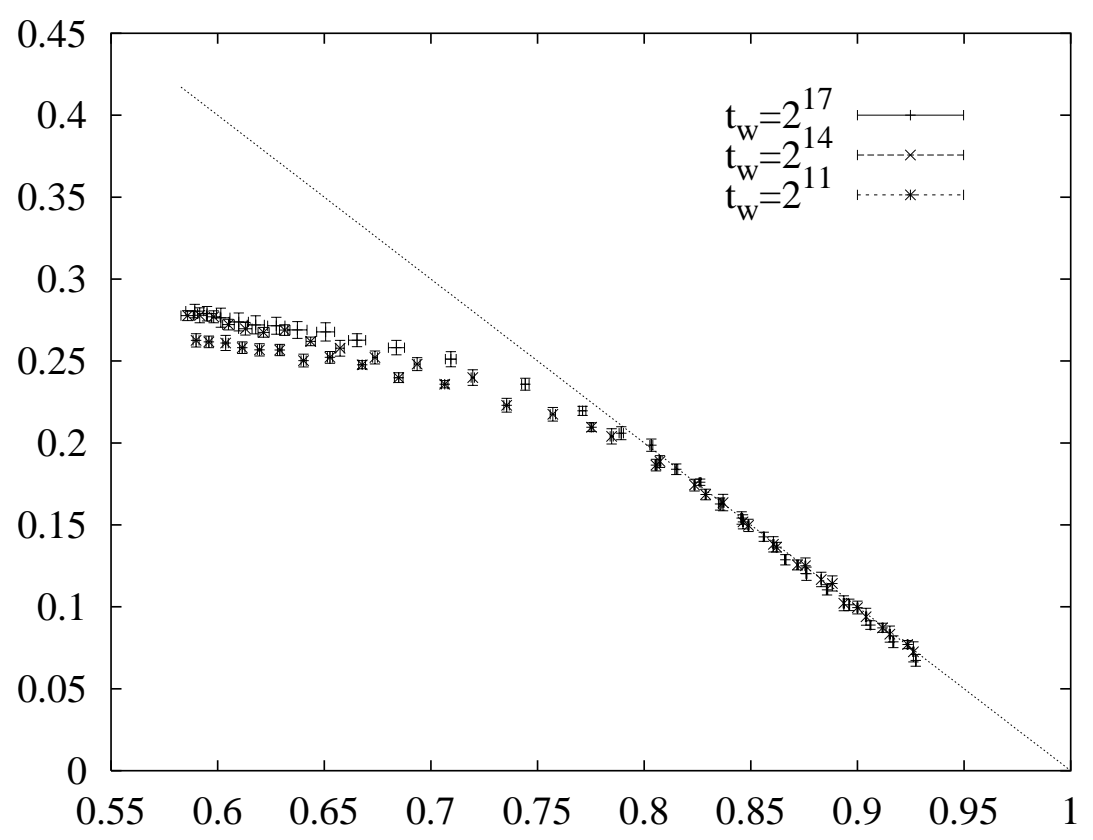

Figure 4: $m_{\mathrm{s}}[\tilde{h}](t) T / \epsilon_{0}$ versus $C\left(t, t_{w}\right)$, with $T=0.75, h_{0}=0.3$ and $\epsilon_{0}=0.06$. The straight line is $1-C$.

From the function $S(C)$ we can get information on the overlap distribution function $P(q)$, through eq. (23). Let us remind which is the prediction for the $S(C)$ assuming the validity of one of the competing theories described in the introduction. The droplet model predicts $P(q)=\delta(q-\hat{q})$ and consequently

$$
S(C)=\left\{\begin{array}{cl}
1-\hat{q} & \text { for } C \leq \hat{q} \\
1-C & \text { for } C>\hat{q}
\end{array}\right.
$$

i.e. there is no dependence of the staggered magnetization on $C\left(t, t_{w}\right)$ in the off-equilibrium regime $(C \leq \hat{q})$, like an ordered ferromagnet [20]. On the other hand the MF like prediction for the overlap distribution [2] $P(q)=\left(1-x_{M}\right) \delta\left(q-q_{\max }\right)+x_{m} \delta\left(q-q_{\min }\right)+\tilde{p}(q)$ (where the support of $\tilde{p}(q)$ belongs to the interval $\left.\left[q_{\min }, q_{\max }\right]\right)$, implies that

$$
S(C)=\left\{\begin{array}{cl}
S(0) & \text { for } C \leq q_{\min } \\
\tilde{s}(C) & \text { for } q_{\min }<C \leq q_{\max } \\
1-C & \text { for } C>q_{\max }
\end{array}\right.
$$

where $\tilde{s}(C)$ is a quite smooth and monotonically decreasing function such that

$$
\tilde{p}(q)=-\left.\frac{\mathrm{d}^{2} \tilde{s}(C)}{\mathrm{d} C^{2}}\right|_{C=q} .
$$

In figure 4 we plot $m_{\mathrm{s}}[\tilde{h}](t) T / \epsilon_{0}$ versus $C\left(t, t_{w}\right)$, with $T=0.75, h_{0}=0.3$ and $\epsilon_{0}=0.06$. The data are the average over 6 samples of a $32^{4}$ system. We can see from figure 4 that, 


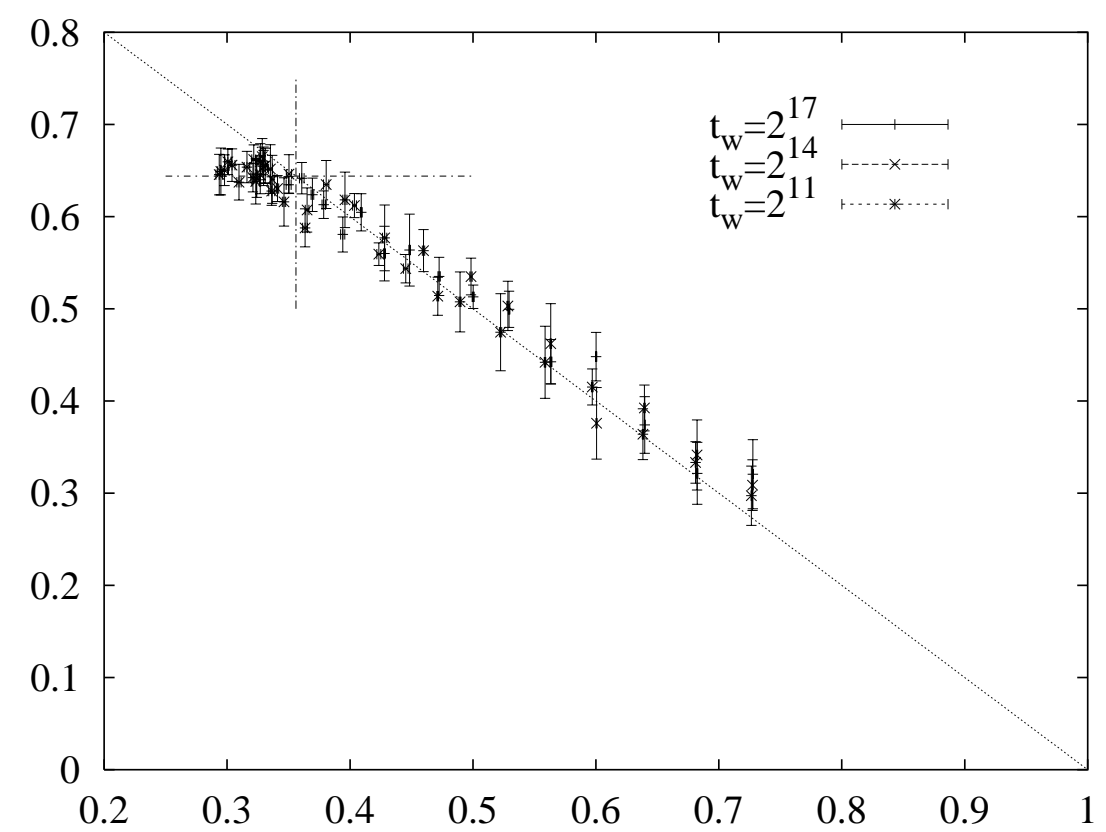

Figure 5: $m_{\mathrm{s}}[\tilde{h}](t) T / \epsilon_{0}$ versus $C\left(t, t_{w}\right)$, with $T=1.5, h_{0}=0.3$ and $\epsilon_{0}=0.02$. The straight line is $1-C$. See text for the short vertical and horizontal lines.

when the data are not on the straight line (FDT or quasi-equilibrium regime), they do not lie on an horizontal line, i.e. they depend on the value of the autocorrelation also in the off-equilibrium regime. So we can conclude that the droplet theory is not able to describe the data in the frozen phase.

Interpreting the data using the MF picture, we deduce from the small curvature of the function $S(C)$ in the region $C \leq q_{\max }$ that $\tilde{p}(q)$ is small. Anyway the existence of two different delta functions in $P(q)$ is clear.

To verify the correctness of the method we present in figure 5 the same kind of plot for a system which is in the paramagnetic phase $\left(T=1.5, h_{0}=0.3\right.$ and $\left.\epsilon_{0}=0.02\right)$. The data stay, as they should, on the equilibrium line $S(C)=1-C$. The few points which leave the straight line are those with the lowest $t_{w}$ and large $t$. This effect is due to the fact that the autocorrelation $C\left(t, t_{w}\right)$ tends to $q_{\text {min }}$ only if the system at time $t_{w}$ has reached equilibrium. On the contrary if the system is in a random configuration at time $t_{w}$ the autocorrelation will tend to zero also in a magnetic field. In our simulation the system is in an intermediate situation and so the lowest value for the autocorrelation with $t_{w}=2^{11}$ is something smaller than $q_{\text {min }}$, while for $t_{w}=2^{17}$ we think that the system is nearly equilibrated and the autocorrelation does not decrease beyond $q_{\text {min }}$. In figure 5 we have reported the value for the equilibrium overlap, $\bar{q}=q_{\min }=q_{\max } \simeq 0.356$, calculated with the annealing runs (vertical line) and the corresponding staggered magnetization (horizontal line). Though it is not very clear from figure 5, we have verified that for every $t_{w}$ the staggered magnetization saturates to the value marked with an horizontal line and that

\footnotetext{
${ }^{7}$ The data can be fitted also with a straight line, whose non-zero inclination is almost $t_{w}$-independent.
} 
for the greater waiting time the autocorrelation tends to the vertical line.

\section{Conclusions}

We have obtained, using off-equilibrium simulations, $q_{\min }, q_{\max }$ and $\bar{q}$ for the four dimensional Gaussian spin glass in presence of a magnetic field finding that in the low temperature region $q_{\min }<\bar{q}<q_{\max }$ according with the predictions of Mean Field Theory.

This result point clearly toward to a phase transition between a spin glass phase with spontaneously broken replica symmetry $\left(q_{\min }<\bar{q}<q_{\max }\right)$ and a phase where the replica symmetry is stable $\left(q_{\min }=\bar{q}=q_{\max }\right)$.

Moreover we have extended the numerical studies of the validity of the fluctuationdissipation theory obtaining that the function that determines the violation depends only on the correlation, as Mean Field theory predicts. We plan in the future to try to link this function $(X)$ with that obtained from the static of the system $(x)$ as it has been done in absence of magnetic field although some indirect evidences of this link has been shown in the present paper.

\section{Acknowledgments}

We acknowledge interesting discussions with E. Marinari and F. Zuliani. J. J. Ruiz-Lorenzo is supported by an EC HMC (ERBFMBICT950429) grant. 


\section{References}

[1] W. L. McMillan, J. Phys. C 17, 3179 (1984). A. J. Bray and M. A. Moore, J. Phys. C 18, L699 (1985). G. J. Koper and H. J. Hilhorst, J. de Physique 49, 429 (1988). D. S. Fischer and D. A. Huse, Phys. Rev. B 38, 386 (1988); Phys. Rev. B 38, 373 (1988).

[2] M. Mézard, G. Parisi and M. A. Virasoro, Spin Glass Theory and Beyond. World Scientific (Singapore 1987).

[3] A. J. Bray and S. A. Roberts, J. Phys. C 13, 5405 (1980).

[4] S. Caracciolo, G.Parisi, S. Patarnello and N. Sourlas, Europhys. Lett. 11, 783 (1990); J. de Physique 51, 1877 (1990). E. R. Grannan and R. E. Hetzel, Phys. Rev. Lett. 67, 907 (1991). J. C. Ciria, G. Parisi, F. Ritort and J. J. Ruiz-Lorenzo, J. de Physique I 3, 2207 (1993). M. Picco and F. Ritort, J. de Physique I 4, 1619 (1994); condmat/9702041.

[5] E. Marinari, G. Parisi and F. Zuliani, cond-mat/9703253.

[6] E. Marinari, G. Parisi and J. J. Ruiz-Lorenzo. "Numerical Simulations of Spin Glass Systems" in "Spin Glasses and Random Fields", edited by P. Young. World Scientific (Singapore 1997). cond-mat/9701016.

[7] G.Parisi, Field Theory, Disorder and Simulations. World Scientific (Singapore 1992).

[8] J. R. L. de Almeida and D. J. Thouless, J. Phys. A: Math. Gen. 11, 983 (1978).

[9] E. Marinari, G. Parisi and J. J. Ruiz-Lorenzo. In preparation.

[10] J.-P. Bouchaud, L. F. Cugliandolo, J. Kurchan, M. Mézard. "Out of equilibrium dynamics in spin-glasses and other glassy systems" in "Spin Glasses and Random Fields", edited by P. Young. Word Scientific (Singapore 1997). cond-mat/9702070

[11] S. Franz and H. Rieger, J. Stat. Phys. 79749 (1995).

[12] E. Marinari, G. Parisi, F. Ricci-Tersenghi and J. J. Ruiz-Lorenzo, cond-mat/9710120.

[13] L. F. Cugliandolo and J. Kurchan, Phys. Rev. Lett. 71, 173 (1993); Philosophical Magazine 71, 501 (1995); J. Phys. A: Math. Gen. 27, 5749 (1994).

[14] S. Franz and M. Mézard, Europhys. Lett. 26, 209 (1994).

[15] A. Baldassarri, L. F. Cugliandolo, J. Kurchan and G. Parisi, J. Phys. A: Math. Gen. 28, 1831 (1995).

[16] C. Battista et al., Int. J. High Speed Comp. 5, 637 (1993).

[17] G. Parisi, F. Ricci-Tersenghi and J. J. Ruiz-Lorenzo, J. Phys. A: Math. Gen. 29, 7943 (1996). 
[18] E. Marinari, G. Parisi, J. J. Ruiz-Lorenzo and F. Ritort. Phys. Rev. Lett 76, 843 (1996).

[19] G. Parisi, P. Ranieri, F. Ricci-Tersenghi and J. J. Ruiz-Lorenzo, J. Phys. A: Math. Gen. 30, 7115 (1997).

[20] A. Barrat, cond-mat/9710069. 